\title{
UV Spectrophotometric Method Development and Validation of Fimasartan Drug and Its Tablet Formulation
} Agrawal Kaushik S *, Gandhi Lokesh R, Bhajipale Nitin S

S.G.S.P.S. Institute of Pharmacy, Kaulkhed, Akola, Maharashtra, India

\begin{abstract}
A B S T R A C T
A novel, safe and sensitive method of Spectrophotometric estimation in UV - region has been developed for the assay of Fimasartan in its tablet formulation. The present study was undertaken to develop and validate a simple, accurate, precise, reproducible and cost effective UV spectrophotometric method for the estimation of Fimasartan bulk and pharmaceutical formulation. The method have been developed and validated for the assay of Fimasartan using Methanol as diluent. Absorption maximum $(\lambda \max )$ of the drug was found to be $240 \mathrm{~nm}$. The quantitative determination of the drug was carried out at $240 \mathrm{~nm}$. The method was shown linear in the mentioned concentrations having correlation coefficient $\mathrm{R}^{2}$ of 0.999 . The recovery values for Fimasartan ranged from 98.74\% to $99.23 \%$.The Percent Relative Standard Deviation of interday and intraday was $0.85 \%$ and $0.75 \%$ respectively. All the parameters of the analysis were chosen according to the International Conference on Harmonisation guideline and validated statistically using Relative Standard Deviation and Percent Relative Standard Deviation. Hence, proposed method was precise, accurate and cost effective. This method could be applicable for quantitative determination of the bulk drug as well as dosage formulation.
\end{abstract}

KEY WORDS: UV Spectrophotometer, Fimasartan, Method Validation, Linearity

A R T I C L E I N F O: Received 03 July 2019; $\quad$ Review Completed 05 Sept 2019; $\quad$ Accepted 15 Sept 2019; $\quad$ Available online 15 Oct. 2019

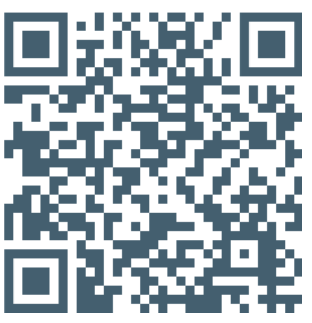

Cite this article as:

Agrawal KS*, Gandhi LR, Bhajipale NS, UV Spectrophotometric Method Development and Validation of Fimasartan Drug and Its Tablet Formulation, Asian Journal of Pharmaceutical Research and Development. 2019; 7(5):26-30

DOI: http://dx.doi.org/10.22270/ajprd.v7i5.576

*Address for Correspondence:

Kaushik Agrawal, S.G.S.P.S. Institute of Pharmacy, Kaulkhed, Akola, Maharashtra, India - 444004

\section{INTRODUCTION}

\section{Spectroscopy Methods}

$\mathrm{I}$ $\mathrm{t}$ is the branch of science dealing with the study of interaction between Electromagnetic radiation and matter. It is a most powerful tool available for the study of atomic and molecular structure/s and is used in the analysis of wide range of samples. Optical spectroscopy includes the region on electromagnetic spectrum between $100 \AA$ and $400 \mu \mathrm{m} .{ }^{1,2}$ The regions of electromagnetic spectrum are shown in Table No. 1.

\section{UV Spectrophotometry}

UV spectrophotometry is one of the most frequently employed techniques in pharmaceutical analysis. It involves measuring the amount of ultraviolet or visible radiation absorbed by a substance in solution. Instrument which measure the ratio, or function of ratio, of the intensity of two beams of light in the UV region are called UV spectrophotometers.

In qualitative analysis, organic compounds can be identified by use of spectrophotometer, if any recorded data is available, and quantitative spectrophotometric analysis is used to ascertain the quantity of molecular species absorbing the radiation. Spectrophotometric technique is simple, rapid, moderately specific and applicable to small quantities of compounds. The fundamental law that governs the quantitative spectrophotometric analysis is the Beer -Lambert law. ${ }^{3}$

Beer's law: It states that the intensity of a beam of parallel monochromatic radiation decreases exponentially with the 
number of absorbing molecules. In other words, absorbance is proportional to the concentration.

Lambert's law: It states that the intensity of a beam of parallel monochromatic radiation decreases exponentially as it passes through a medium of homogeneous thickness. A combination of these two laws yields the Beer-Lambert law.

Beer-Lambert law: When beam of light is passed through a transparent cell containing a solution of an absorbing substance, reduction of the intensity of light may occur. Mathematically, Beer- Lambert law is expressed as $A=a b c$ Where, $A=$ absorbance or optical density

$a=$ absorptivity or extinction coefficient

$\mathrm{b}=$ path length of radiation through sample $(\mathrm{cm})$

$\mathrm{c}=$ concentration of solute in solution.

Both $\mathrm{b}$ and $\mathrm{a}$ are constant so $\mathrm{a}$ is directly proportional to the concentration $\mathrm{c}$

When $\mathrm{c}$ is in $\mathrm{gm} / 100 \mathrm{ml}$, then the constant is called A $(1 \%, 1$ $\mathrm{cm})$

$$
\mathrm{A}=\mathrm{A}(1 \% / 1 \mathrm{~cm}) \mathrm{bc}
$$

Quantification of medicinal substance using spectrophotometer may carried out by preparing solution in transparent solvent and measuring it's absorbance at suitable wavelength. The wavelength normally selected is wavelength of maximum absorption $(\lambda \max )$, where small error in setting the wavelength scale has little effect on measured absorbance.

Table 1: Regions of electromagnetic spectrum

\begin{tabular}{|c|c|}
\hline Region & Wavelength \\
\hline Far ultraviolet & $10-200 \mathrm{~nm}$ \\
\hline Near ultraviolet & $200-400 \mathrm{~nm}$ \\
\hline Visible & $400-750 \mathrm{~nm}$ \\
\hline Near infrared & $0.75-2.2 \mu \mathrm{m}$ \\
\hline Mid infrared & $2.5-50 \mu \mathrm{m}$ \\
\hline Far infrared & $50-1000 \mu \mathrm{m}$ \\
\hline
\end{tabular}

\section{INTRODUCTION TO FIMASARTAN}

Fimasartan is a non-peptide angiotensin II receptor antagonist used for the treatment of hypertension and heart failure. Through oral administration, fimasartan blocks angiotensin II receptor type 1 ( $\mathrm{AT}_{1}$ receptors), reducing prohypertensive actions of angiotensin II, such as systemic vasoconstriction and water retention by the kidneys. Concurrent administration of fimasartan with diuretic hydrochlorothiazide has shown to be safe in clinical trials. Fimasartan is being marketed in India under the brand name of Fimanta and Fimagen through Ajanta Pharma Ltd. ${ }^{4,5}$

\section{Description}

IUPAC name: 2-n-butyl-5-dimethylamino-

thiocarbonylmethyl -6- methyl-3, pyrimidin-4(3H).

(Fig. 1)

Fimasartan is a non-peptide angiotensin II receptor antagonist (ARB) used for the treatment of hypertension and heart failure. Concurrent administration of fimasartan with diuretic hydrochlorothiazide has shown to be safe in clinical trials. Through oral administration, fimasartan blocks angiotensin II receptor type 1 ( $\mathrm{AT}_{1}$ receptors), reducing prohypertensive actions of angiotensin II, such as systemic vasoconstriction and water retention by the kidneys. ${ }^{14,15,16}$

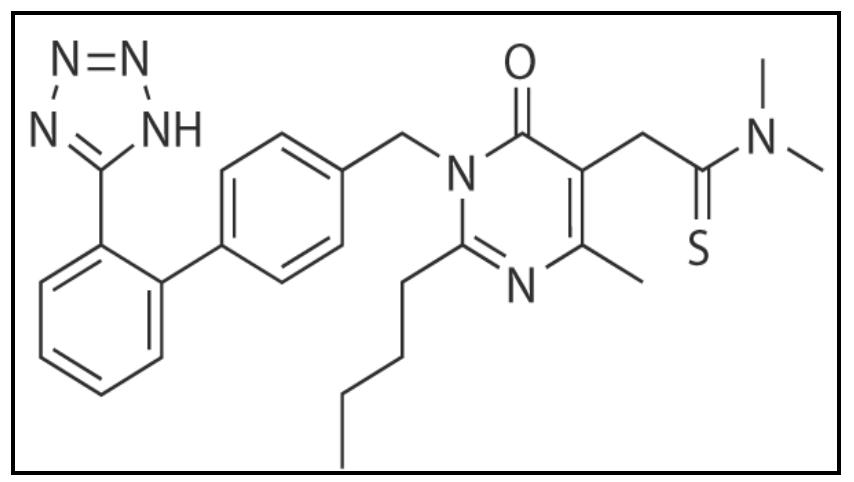

Figure 1: Structure of Fimasartan

\section{Mechanism of action}

Fimasartan acts on the kidney's rennin-angiotensin cascade, which begins when renin release from the kidney causes the breakdown of angiotensinogen into angiotensin $\mathrm{I}$. Angiotensin-converting enzyme (ACE) then catalyzes the reaction that forms angiotensin II, which acts on $\mathrm{AT}_{1}$ receptors on the blood vessels, heart, and kidneys. On blood vessels, the $\mathrm{AT}_{1}$ receptor is coupled to an intracellular pathway that causes vasoconstriction of blood vessels. In blocking the $\mathrm{AT}_{1}$ receptor, fimasartan inhibits vasoconstriction, favouring vasodilation. ARBs like fimasartan have also shown to be protective against stroke, myocardial infarction, and heart failure.

\section{Pharmacology}

Fimasartan is rapidly absorbed and has minimal accumulation in the body 7 days after administration. Fimasartan possesses a half-life of 9 to 16 hours, appropriate for daily dosing. Fimasartan was found to be effective in fasted or fed states, with a variety of dosing regimens. Fimasartan was found mostly in unmetabolized form in the plasma and in bile excretions. Urinary elimination of the drug was low, at less than $3 \% 24$ hours after administration, entailing that the fimasartan does not undergo renal excretion. ${ }^{15,16}$

\section{METHOD VALIDATION}

Validation is concerned with assuring that a measurement process produces valid measurements. Results from method validation can be used to judge the quality, reliability and consistency of analytical results. It is an integral part of any good analytical practice. A measurement process producing valid measurements for an intended application is fit for purpose.

Analytical methods need to be validated or revalidated, before their introduction into routine use; whenever the conditions change for which the method has been validated.

ICH guidelines (ICH Q2R1) for analytical procedure and validation 
The analytical procedure refers to the way of performing the analysis. It should describe in detail the steps necessary to perform each analytical test. This may include but is not limited to: the sample, the reference standard and the reagents preparations, use of the apparatus, generation of the calibration curve, use of the formula for the calculation. ${ }^{17,19}$

\section{Types of analytical procedures to be validated}

The objective of the analytical procedure should be clearly understood since this will govern the validation characteristics which need to be evaluated.

- Accuracy

- Precision

- Repeatability

- Specificity

- Linearity

- Range

- Robustness

- Ruggedness

\section{AIM OF PRESENT WORK}

This work deals with the validation of the developed method for the assay of Fimasartan from its dosage form (tablets). Hence, the method can be used for routine quality control analysis and also stability. The aim and scope of the proposed work are as under:

- To develop suitable spectrophotometric method for assay of Fimasartan tablet.

- Perform the validation for the method.

\section{EXPERIMENTAL}

\section{Materials}

Fimasartan standard was provided by Ajanta Pharma Pvt. Ltd. (India). Fimagen tablets containing $60 \mathrm{mg}$ Fimasartan was obtained from market. Analytical grade methanol obtained from Spectrochem Pvt. Ltd., Mumbai (India).

\section{Diluent Preparation}

Methanol used as a diluent.

\section{Standard Preparation}

$1 \mathrm{mg}$ drug was dissolved in $10 \mathrm{ml}$ methanol and was shaken well. Then methanol was added to it to adjust the volume up to $100 \mathrm{ml}$. From that, dilutions of concentration 2, 4, 6, 8, 10, $12,14,16,18,20 \mu \mathrm{g} / \mathrm{ml}$ was prepared by using diluent.

\section{Test Preparation}

Weigh 20 tablets and powdered. Powdered tablet equivalent to $60 \mathrm{mg}$ of Fimasartan was weighed and taken into $100 \mathrm{ml}$ volumetric flask then $50 \mathrm{ml}$ of methanol was added and shaken well to dissolve it after that methanol was added to adjust the volume up to $100 \mathrm{ml}$. From this solution required dilutions are prepared.

\section{Instrumentation}

UV-Visible double beam spectrophotometer with matched quartz cells.

Model: UV - 1800 (Shimadzu)

\section{Selection of Wavelength}

Scan standard solution in UV spectrophotometer between $200 \mathrm{~nm}$ to $400 \mathrm{~nm}$ on spectrum mode, using diluents as a blank. Fimasartan shows $\lambda \max$ at 240. (Fig. 2)

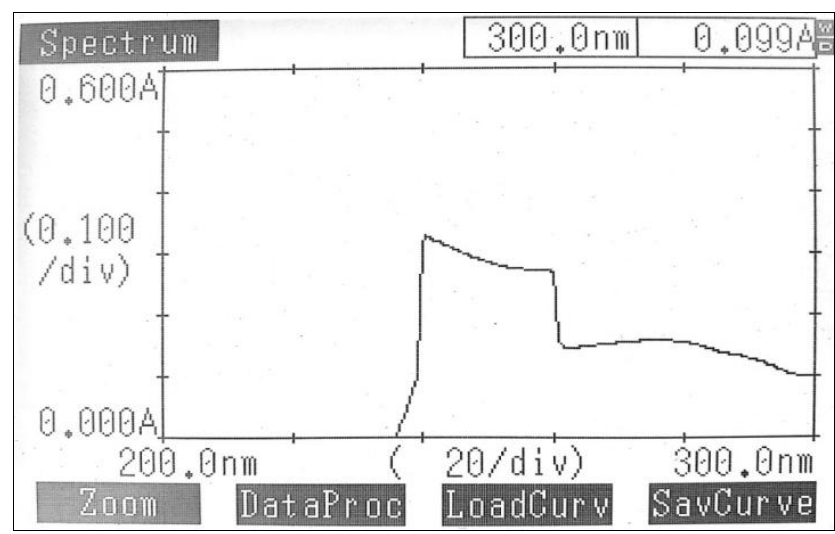

Figure 2: Graph of UV $\lambda \max$

\section{METHOD VALIDATION}

Linearity: Fresh aliquots were prepared from standard stock solution ranging from $2-20 \mu \mathrm{g} / \mathrm{ml}$ and the absorbance values of each was recorded at $240 \mathrm{~nm}$ for this method using Methanol as blank. The response was found to be linear in the investigation concentration range. (Table No. 2, Fig. 3).

Table 2: Concentration versus Absorbance table for Linearity Study

\begin{tabular}{|c|c|}
\hline Conc. $($ ppm $)$ & Absorbance \\
\hline 0 & 0 \\
\hline 2 & 0.184 \\
\hline 4 & 0.401 \\
\hline 6 & 0.562 \\
\hline 8 & 0.729 \\
\hline 10 & 0.905 \\
\hline 12 & 1.108 \\
\hline 14 & 1.252 \\
\hline 16 & 1.412 \\
\hline 18 & 1.582 \\
\hline 20 & 1.786 \\
\hline
\end{tabular}

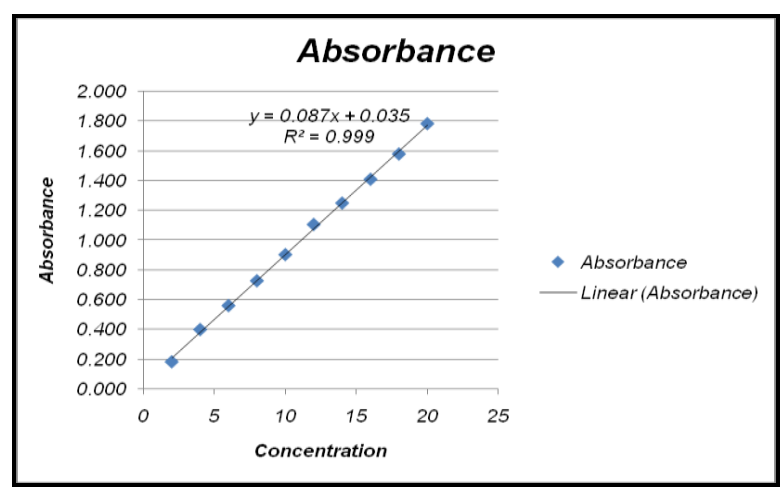

Figure 3: Linearity Curve for Fimasartan 
Precision: In intraday study, concentration of replicates of drug was calculated on the same day for three times. In inter-day study the concentration of drug were calculated on three successive days which expresses the laboratory variation in different days. In both intra and inter day precision study for the methods \%RSD was calculated. (Table No. 3).

Accuracy: Accuracy of the developed method was confirmed by performing recovery studies at three different concentration ranges $80 \%, 100 \%, 120 \%$ each one in triplicate. From the recovery studies it was clear that the method is very accurate for quantitative estimation of tablet as the statistical results were within the acceptance range. (Table No. 4).

Table 3: Evaluation data of precision study

\begin{tabular}{|c|c|c|}
\hline Sample No. & \multicolumn{2}{|c|}{ \% Assay } \\
\hline SET & Intraday & Interday \\
\hline 1 & 101.8 & 100.4 \\
\hline 2 & 101.7 & 98.1 \\
\hline 3 & 101.1 & 99.2 \\
\hline 4 & 101.5 & 100.1 \\
\hline 5 & 100.7 & 99.0 \\
\hline 6 & 99.8 & 99.8 \\
\hline Mean & 101.1 & 99.4 \\
\hline SD & 0.76 & 0.84 \\
\hline \% RSD & 0.75 & 0.85 \\
\hline
\end{tabular}

Table 4: Evaluation data of accuracy study

\begin{tabular}{|c|c|c|c|c|}
\hline $\begin{array}{l}\text { \% Recovery } \\
\text { Level }\end{array}$ & \% Recovery & $\begin{array}{l}\text { Mean \% } \\
\text { Recovery }\end{array}$ & $S D$ & $\% R S D$ \\
\hline \multirow{3}{*}{$50 \%$} & 98.83 & \multirow{3}{*}{98.83} & \multirow{3}{*}{0.0450} & \multirow{3}{*}{0.0455} \\
\hline & 98.78 & & & \\
\hline & 98.87 & & & \\
\hline \multirow{3}{*}{$100 \%$} & 98.74 & \multirow{3}{*}{98.76} & \multirow{3}{*}{0.0152} & \multirow{3}{*}{0.0154} \\
\hline & 98.76 & & & \\
\hline & 98.77 & & & \\
\hline \multirow{3}{*}{$125 \%$} & 99.23 & \multirow{3}{*}{99.22} & \multirow{3}{*}{0.0100} & \multirow{3}{*}{0.0100} \\
\hline & 99.21 & & & \\
\hline & 99.22 & & & \\
\hline
\end{tabular}

Robustness: It should show the reliability of an analysis with respect to deliberate variations in method parameters. The result of robustness study of the developed assay method was established. The result shown that during all variance conditions, assay value of the test preparation solution was not affected and it was in accordance with that of actual. System suitability parameters were also found satisfactory.
Ruggedness: Ruggedness of the method was determined by carrying out the analysis by two different analysts and the respective absorbance was noted. The result was indicated by $\%$ RSD.

\section{RESULTS AND DISCUSSION}

The developed method was found to be precise as the $\%$ RSD values for intra-day and inter-day were found to be less than $1 \%$. Good recoveries (98.74\% to $99.23 \%$ ) of the drug were obtained at each added concentration, which indicates that the method was accurate. The method was also found to be robust and rugged as indicated by the $\%$ RSD values which are less than $1 \%$.

\section{CONCLUSION}

The present analytical method was validated as per ICH guideline and it meets to specific acceptance criteria. It is concluded that the analytical method was specific, precise, linear, accurate, robust and having stability indicating characteristics. The present analytical method can be used for its intended purpose.

\section{ACKNOWLEDGEMENTS}

We are thankful to the Principal of S. G. S. P. S. Institute of Pharmacy College for his constant encouragement for carrying out this work. We would like to thank Ajanta Pharma Pvt Ltd. for providing the gift sample of Fimasartan.

\section{REFERENCE}

1. Willard-Hobart H, Merritt Jr Lynne L, Dean John A. Instrumental Methods of Analysis, $5^{\text {th }}$ edition, Von Nostrand, University of Michigan, 1966; 43(9):506

2. Chatwal G R, Anand S. Instrumental Methods of Chemical Analysis, Himalaya Publishing House, New Delhi, 2005

3. Beckett AH, Stenlake JB. Practical Pharmaceutical chemistry, $4^{\text {th }}$ edition, CBS Publishers and distributors, New Delhi, 2007; 275-278.

4. Pradhan A, Gupta V, Sethi R. Fimasartan: A new armament to fight hypertension, Journal of Family Med Prim Care 2019; 8:2184-8

5. Shin KH, et al. The effect of the newly developed angiotensin receptor II antagonist fimasartan on the pharmacokinetics of atorvastatin in relation to OATP1B1 in healthy male volunteers, Journal of Cardiovascular Pharmacology, 2011; 492-499.

6. Guideline IHT. Validation of Analytical Procedures: Text and Methodology, Q2 (R1), Current Step 4 Version, Parent Guidelines on Methodology, International Conference on Harmonisation, Geneva, Switzerland, 2005

7. Sica D. Rationale for fixed-dose combinations in the treatment of hypertension. Drugs 2002; 443-462.

8. Sohn Y. Crystal forms of an angiotensin II receptor antagonist BRA657, Journal of Thermal Analysis and Calorimetry 2007; 799-802.

9. Chi YH, Lee H, Paik SH. Safety, tolerability, pharmacokinetics and pharmacodynamics of fimasartan following single and repeated oral administration in the fasted and fed states in healthy subjects, Journal of Cardiovascular Drugs 2011; 11:335-346.

10. Kim JW, Yi S, Kim TE. Increased systemic exposure of fimasartan, an angiotensin II receptor antagonist, by ketoconazole and rifampicin, Journal of Clinical Pharmacology 2013; 53:75-81.

11. Yi S, Kim J, Kim T. Effect of multiple doses of fimasartan, an angiotensin II receptor antagonist, on the steady-state pharmacokinetics of digoxin in healthy volunteers, Journal of Clinical Pharmacology 2011; 49:321-327.

12. Yi S, Kim T-E, Yoon SH. Pharmacokinetic interaction of fimasartan, a new angiotensin II receptor antagonist, with amlodipine in healthy volunteers. Journal of Cardiovascular Pharmacology 2011; 57:682689. 
13. Jeon H, Lim KS, Shin K-Hl. Assessment of the drug-drug interactions between fimasartan and hydrochlorothiazide in healthy volunteers. Journal of Cardiovascular Pharmacology 2012; 59:84-91.

14. Ryu S, et al. Fimasartan, anti-hypertension drug, suppressed inducible nitric oxide synthase expressions via nuclear factor-kappa B and activator protein-1 inactivation. Biological and Pharmaceutical Bulletin, 2013; 36(3):467-74.

15. Chi YH, et al. Pharmacological characterization of BR-A-657, a highly potent nonpeptide angiotensin II receptor antagonist. Biological and Pharmaceutical Bulletin. 2013; 36(7):1208-15.

16. Lee HW, et al. Effect of age on the pharmacokinetics of fimasartan (BR-A-657), Expert Opin Drug Metab Toxicology. 2011; 7(11):133744.
17. International Conference on Harmonisation, Q2B Validation of Analytical Procedures-Methodology, Consensus Guidelines, ICH Harmonized Tripartite Guidelines, 1996

18. William Foye, Thomas L Lemke, David A Williams, Williams \& Wilkins, Principles of Medicinal Chemistry, $4^{\text {th }}$ edition, 1996; 544-545.

19. H. Guenzler, Verlag Wegscheider, Validation of Analytical Methods, Accreditation and Quality Assurance in Analytical Chemistry, Berlin, 1996.

20. Phillips PA. Interaction between endothelin and angiotensin II Clinical and Experimental Pharmacology and Physiology, 1999; 26(7): 517-518. 